\title{
Imaging of the Arp 220 OH Megamasers with VLBI
}

Colin J. Lonsdale

MIT Haystack Observatory, Westford MA 01886, U.S.A.

P. J. Diamond

National Radio Astronomy Observatory, Socorro NM 87801, U.S.A.

Carol J. Lonsdale

Infrared Processing and Analysis Center, Pasadena CA 91125, U.S.A.

H. E. Smith ${ }^{1}$

University of California San Diego, San Diego CA 92093, U.S.A.

Abstract. Global VLBI imaging of the luminous IR galaxy Arp 220 reveals four major emission regions in the $1667 \mathrm{MHz}$ line, each with complex spatial and velocity structure showing intriguing symmetries, suggestive of an AGN-related phenomenon. The continuum emission consists of about a dozen sub-mJy unresolved features which appear unrelated to the maser emission, and are interpreted as radio supernovae in an ongoing nuclear starburst.

The prototype $\mathrm{OH}$ megamaser is found in the powerful IR galaxy Arp 220 (IC 4553), and exhibits the classical properties of a double-peaked line profile a few hundred $\mathrm{km} / \mathrm{s}$ wide. The favored interpretation of these masers has been that a low-gain masing screen distributed over a region a few hundred parsecs in size amplifies nuclear continuum emission from the galaxy. Recently, it was demonstrated that much of the $\mathrm{OH}$ megamaser emission in Arp 220 arises in physically compact regions, on scales of a few parsecs.

We used a 17-station global VLBI array in November 1994 to observe Arp 220 and three other $\mathrm{OH}$ megamaser sources. The Arp 220 data were correlated in Socorro in April 1996, and reduced using AIPS. We were able to detect a strong, compact maser feature on baselines to all telescopes at all times, and thus were able to phase-reference the entire dataset to this feature. The resulting noise-limited spectral line and continuum images are shown below.

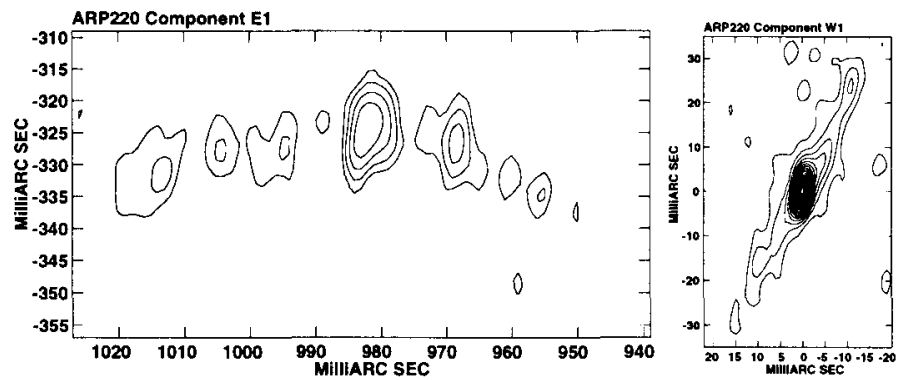

Figure 1. Line image of elongated features in each nucleus of Arp 220

Each of the two nuclei in Arp 220, 1 arcsec apart, contains two main maser components, one highly elongated and one more amorphous. In each case the elongated component displays a striking symmetry in that a compact, spectrally broad maser spot accurately bisects the overall structure. In these central

${ }^{1}$ Also Infrared Processing and Analysis Center, Pasadena CA 91125, U.S.A. 
features, there is evidence for a sharp velocity gradient perpendicular to the elongation of the overall structure. All the compact masers show high brightness temperatures with no associated continuum emission, strongly suggesting maser saturation. In addition, the physical compactness argues for collisional, rather than IR pumping because any IR pump source must be very extended.

It is possible that the symmetric linear features seen in each nucleus represent material that is shock-heated and collisionally pumped in response to a newly-formed AGN nucleus. The central, spectrally broad feature with the transverse velocity gradient might be identified with an accretion-disk/torus orbiting a central mass of order $10^{8} \mathrm{M}_{\odot}$, while the rest of the linear structure might trace disturbances due to the passage of an unseen pair of jets.

That an active starburst is proceeding in the nucleus of Arp 220 appears almost certain, however, in light of the continuum image. This shows roughly a dozen sub-mJy unresolved $(\leq 0.15 \mathrm{pc})$ point sources, which perfectly fit a model involving bright radio supernovae within a starbursting volume of 50 by $150 \mathrm{pc}$ in $\operatorname{Arp} 220$.

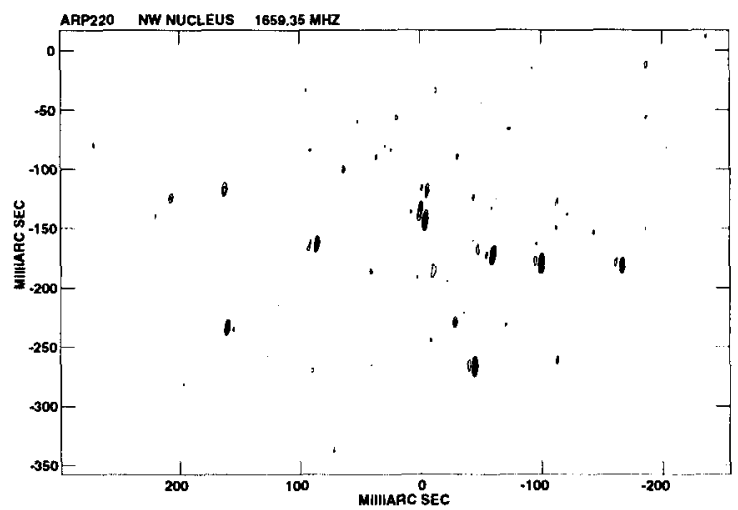

Figure 2. Continuum image of the western nucleus of Arp 220. Each source in this image is thought to be a radio supernova of radio power comparable to that of $\mathrm{SN} 1986 \mathrm{~J}$. The eastern nucleus shows similar, but fewer and weaker, point sources.

Ultraluminous IR galaxies, of which Arp 220 is the prototype example, represent a crucial stage in the process of galaxy collisions and mergers, which are widely suspected to be responsible for the formation of both quasars and elliptical galaxies. By probing the heavily obscured nuclear regions at wavelengths where they are transparent, we have uncovered compelling evidence for an ongoing intense starburst capable of supplying much, if not all, of the enormous bolometric luminosity of the galaxy. At the same time we have redefined the characteristics of the $\mathrm{OH}$ megamaser phenomenon, and discovered tantalizing suggestions of nascent $A G N$ activity in each of the two merging nuclei. It seems likely that our VLBI investigations have for the first time afforded us a clear view of an important and hitherto unobservable stage in galaxy evolution, from which both elliptical galaxies and quasars may emerge.

Acknowledgments. The National Radio Astronomy Observatory is a facility of the National Science Foundation, operated under a cooperative agreement by Associated Universities, Inc.. HES acknowledges receipt of NSF grant AST93-19895 to UCSD. 